\title{
Intraoperative neurophysiology is here to stay
}

\author{
Francesco Sala
}

Received: 24 January 2010 / Accepted: 25 January 2010/Published online: 24 February 2010

(C) Springer-Verlag 2010

\section{Preface}

The 2010 February and April issues of Child's Nervous System offer to the readers two very timely focus sessions in the field of "neuromonitoring". This was the topic of a symposium held in Cape Town in October 2008-as a satellite event of the 36th Meeting of the International Society for Pediatric Neurosurgery-aimed to cover the various techniques available to neurosurgeons for keeping under control the brain and the spinal cord, both in the operating room and the intensive care unit. What emerged from that symposium is presented in these focus sessions, where international experts in traumatic brain injury (see the Editorial of Dr. Figaji in the February issue of Child's Nervous System) and intraoperative neurophysiology address the aspects of these disciplines relevant to pediatric neurosurgery.

\section{Introduction}

Intraoperative neurophysiology (IN) has emerged over the last decade, as one of the main avenues of progress within neurosurgery. The idea of applying techniques traditionally used in clinical neurophysiology within the neurosurgical operating room is not novel. Somatosensory (SEPs) and brainstem auditory evoked potentials (BAEPs) have been used for many years during neurosurgical procedures $[5,7]$. Direct mapping of the exposed cortex to localize motor and

\section{F. Sala $(\square)$}

Section of Neurosurgery, Department of Neurological and Visual Sciences, University Hospital,

Piazzale Stefani 1,

37124 Verona, Italy

e-mail: francesco.sala@univr.it language areas dates back to the end of the nineteenth century [4]. In the recent past, there has been a renewed drive toward the implementation of these techniques, thanks to the advent of new methods, such as motor evoked potentials (MEPs). Recording MEPs under general anesthesia [24] has allowed for an increase in the indications for IN and has also expanded clinical research in brain and especially in spinal cord surgery.

Intraoperative neurophysiology is aimed at either identifying functional structures which cannot be recognized purely on the basis of anatomical landmarks (mapping) or to continuously assess the functional integrity of neural pathways which can be injured during surgery (monitoring). The "Why, When and How" regarding the use of IN in pediatric neurosurgery has been the topic of a review which appeared in Child's Nervous System in 2002 [22]. The rationale and indications for IN have not substantially changed since then. Methodologies have to some extent evolved, although the basic principles of monitoring and mapping techniques remain the same. Nevertheless, IN has undoubtedly become more and more accessible and extensively used than it has been in the past. The growing interest for this discipline is well documented by the increasing number of publications in this field, both in the form of peer-reviewed papers and edited books as well as by the number of scientific meetings dedicated to intraoperative neurophysiologic monitoring.

There are scientific societies, such as the American Society for Neurophysiological Monitoring and the International Society for Intraoperative Neurophysiology, whose members are dedicated full- or part-time to IN in their daily practice. These and other scientific societies are now facing the need to establish guidelines, credentials, and training requirements for the practice of IN [16]. The spectrum of expertise and advancements in this field varies quite 
considerably across different countries, but there is no doubt that the driving force is not limited to Europe, North America, and Japan and involves, to a different extent, virtually all five continents.

\section{Intraoperative neurophysiology and the neurosurgeon}

Intraoperative neurophysiology is favored by many neurosurgeons nowadays, but this has not always been the case. In 1994, Dr. Leonard Malis, a master of modern neurosurgery, wrote that: "Monitoring has the same place as training wheels on a bicycle; they are of considerable use for the learning youngster, yet perhaps are a detriment to the skilled cyclist because of increase in time expended and in cost" [14]. At that time, MEP monitoring was in its infancy and most of IN was based on SEPs and BAEP. Falsenegative results - namely a patient waking up from anesthesia with a motor deficit in spite of intraoperatively preserved SEPs - could occur, and this detracted from the reliability of IN [9, 12]. Moreover, neuromonitoring was erroneously considered useful merely for predicting the outcome but not for preventing neurological deficits and this also contributed to its lack of popularity among neurosurgeons. The feeling of "wasting time" when performing IN techniques is still a concern for a number of neurosurgeons, as well as the idea that IN sometimes unjustifiably hinders rather than facilitates the removal of a tumor.

In analogy to the introduction of the operating microscope in the 1970s - far from being widely accepted at that time- IN had to find its way to become welcome in the operating room. The idea of a different professional figure (namely a neurologist or clinical neurophysiologist) working hand in hand with the neurosurgeon in the operating room and advising him whether or not his surgical strategy was impairing the well-being of the nervous system was (and still is) something not readily acceptable to neurosurgeons. The classical reply to the neurophysiologist when a warning message was passed to the neurosurgeon often sounded like: "I didn't do anything, maybe you should check your electrodes first..." Neurophysiological feedback was generally welcome as long as it reassured the neurosurgeon that everything was going well ("Am I doing OK? Are your evoked potentials stable?..."), but was not so well-received whenever there was a change in the evoked potentials that would imply the need to halt or even abandon surgery. Transcranial electrical stimulation for motor evoked potential monitoring sometimes induces muscle twitches that can be bothersome when working under magnification. This has also been a concern to some neurosurgeons.

This reluctance to use IN has significantly changed in the past decade, together with an increasing acknowledgement of the reliability and value of IN in our practice. Good neurological outcome in clinical series where IN has been used and historical control studies where monitored patients did better than those operated on without neurophysiologic monitoring contribute in giving credit to IN.

Yet, some resistance to the use of IN still exists nowadays and relies mainly on the criticism that the use of IN is not "evidence-based".

\section{Is it evidence-based?}

There is little discussion about the fact that IN is not based on class I evidence. Yet, we may ask ourselves what level of evidence we do need to justify the use of a certain medical or surgical treatment. In this regard, two comments are imperative.

First, there is a growing concern about the true level of evidence in evidence-based medicine (EBM), and this is due to the conflict of interest in biomedical research. About $75 \%$ of the clinical trials published in highly ranked medical journals are industry funded, with two thirds of these trials conducted by for-profit research companies rather than academic medical centers [1]. It has therefore been suggested that EBM sometimes has more to do with institutional costs and profits than with the patient's health. Therefore, while recognizing that EBM is supposedly motivated by the desire to provide the best and most modern care available, we have to take EBM with a pinch of salt and be cautious in uncritically grounding our clinical practice on EBM studies alone [13].

Second, although the level of evidence for the benefit of IN is limited to class II and class III studies, it should be recognized that the same level of evidence applies to most of our clinical practice within neurosurgery. From the surgical treatment of acute spinal cord injuries $[3,25]$ to that of aneurysms [19, 26], traumatic brain injury [21], and benign brain tumors [2], even the more accredited analyses (such as Cochrane reviews) fail to demonstrate class I evidence. So, after all, we have to admit that the level of EBM in IN is not worse than that found in neurosurgery generally.

It is very unlikely that class I studies will ever occur in the field of IN. There are at least two reasons for this. First, the likelihood of preventing a neurological deficit using IN is so high for certain pathologies (for example spinal cord tumors) that a controlled study where patients are randomly assigned to a control group or a monitored group would be unethical and unacceptable to patient and surgeon alike $[6,18]$.

Second, the incidence of severe and permanent neurological complications for standard pediatric neurosurgical procedures is quite low. Thus, IN would aim at further reducing a number that is already small to begin with. A power calculation study would therefore predict that the 
number of patients needed for such a study would surpass the number of patients enrolled in most single-institution and, most likely, multicenter studies. For example, in order to demonstrate within a randomized study that evoked potential monitoring reduces the incidence of paraplegia during spine surgery from $2 \%$ to $0.5 \%$, one would require about 860 patients per group.

We should therefore accept that in the future, the benefit of IN will continue to be based on good clinical outcomes, historical control studies, and cost-benefit evaluations.

One of the concerns relating to guidelines in the field of IN is related to their medicolegal implications. Although some reports suggest that the courts are slow in applying new clinical practice guidelines in their decision-making processes [20], it is worthwhile to anecdotally recall a case in which a patient sustained paraplegia after scoliosis surgery, and the plaintiffs' expert supported the view that motor pathway monitoring was considered the standard of care. Paradoxically, at that time, there was no Food and Drug Administration-approved equipment in the USA for MEP monitoring [17].

\section{Cost-benefits of intraoperative neurophysiology}

A few papers have addressed the issue of costs and benefits in neuromonitoring [10,11, 15, 23]. A detailed evaluation of the costs of IN should include not merely those of the equipment and disposables (electrodes, probes, etc.) but also that of training and remuneration for the monitoring personnel. From a purely economic standpoint, benefits of IN generally include the avoidance of neurological complications in terms of both rehabilitation costs and economic compensation for malpractice lawsuits. It is noticeable that human suffering is rarely taken into account in cost/benefit analyses while it probably should [15]. Although most of the papers published conclude that benefits outweigh costs, it is obvious that the cost for each monitored procedure decreases along with the increasing number of cases monitored at a single institution.

The cost-benefit argument is not trivial as it is the one that influences hospital administrators when they decide where to invest in Health Care. The impression is that Health Care Systems are increasingly sensitive to the value of intraoperative neurophysiology as to decrease morbidity is one of the goals. The main challenge remains the discrepancy between the increasing demand and a limited offering. IN equipment is not particularly expensive when compared to the other tools that we use daily in the operating room. An average 32-channel system ranges between 60 and 90,000 euros. In the majority of Western countries, this does not represent an insurmountable cost. Therefore, the true limitation is lack of human resources: dedicated personnel and training. Small neurosurgical units with a limited number of elective cases where IN would be needed cannot justify the costs of hiring a dedicated intraoperative neurophysiologist or the costs of providing appropriate training to a staff member. This has encouraged the development of private companies which provide trained neurophysiologists or technicians ready to travel from one hospital to another to perform neuromonitoring. So-called "remote monitoring" is another way to obviate the lack of trained personnel: a neurophysiologist monitoring, via teleconference, the evoked potential traces recorded in an operating room hundreds of miles away. This, which may sound like "science fiction medicine", is being practiced in reality in some countries like the USA. The debate around remote neuromonitoring is much in vogue, as it is the debate around credentials and training. Who is entitled to perform IN, what is an appropriate curriculum, and what are the appropriate credentials remain a matter of debate and the answer is yet to come.

\section{The hype cycle of intraoperative neurophysiology}

In conclusion, we may see the development of IN during the past $10-15$ years being well represented by a so-called hype cycle [8]. A hype cycle is a graphic representation of the maturity, adoption, and business application of specific technologies which also has some analogy with the advent and development of new treatments and tools in health care (Fig. 1).

A hype cycle comprises five phases:

1. Technology trigger: This is the first phase, a breakthrough that occurs when a new product is launched on the market generating interest and attraction. In our setting, this dates back to the implementation of IN techniques in the mid- and late-1990s.

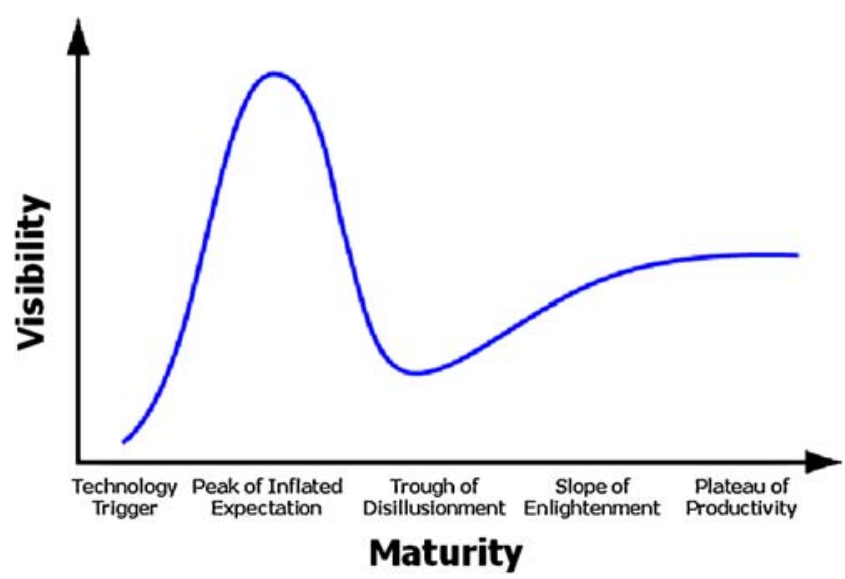

Fig. 1 The hype cycle of intraoperative neurophysiology (see text for details) 
2. Peak of inflated expectations: This is what happens when overenthusiasm and unrealistic expectations are generated. In this phase, successful applications and failures of a technology occur. We may apply this to the time when too much expectation was placed on SEPs followed by the first false-negative results appearing in the literature.

3. Trough of disillusionment: This is what happens when expectations are not met and technologies become unfashionable. This may correspond to the time, immediately before the advent of MEPs, when the limitations of SEPs became evident but no alternatives were available. A similar disillusionment is experienced even today whenever we expect IN to be more reliable in specificity and sensitivity.

4. Slope of enlightenment: This is the phase of maturity, when in spite of some failures, research continues to grow, identify reliable goals, and extend applications. The advent of intraoperative MEPs represented a real breakthrough in this regard, together with major awareness of the value and limitations of different IN techniques.

5. The plateau of productivity: This corresponds to the phase where the benefits of a technology become widely demonstrated and accepted, leaving behind prejudices. Today, IN is experiencing a plateau of productivity in tertiary care hospitals and academic institutions where it is performed according to both the highest professional level and standards of care. It is hoped that in our patients' best interest, IN will become available on a larger scale. As neurosurgeons, there is no valid reason to reject the support that IN can provide to us. IN is not aimed at replacing a deep knowledge of neuroanatomy nor can it surrogate a lack of clinical judgment when facing challenging situations in the operating room. However, it does represent one more tool in our hands which we can use "ad hoc" to improve our results and make neurosurgery safer.

A number of colleagues contributed to this focus session on intraoperative neurophysiology. Dr. Abbott provided a thoughtful view on the pediatric neurosurgeon's perspective. Dr. Sloan offered an updated review on the critical topic of pediatric neuroanesthesia for intraoperative neurophysiological monitoring. Besides these introductory papers, from brain tumor and epilepsy surgery in eloquent brain areas (Dr. $\mathrm{Ng} /$ Dr. Rutka, Dr. Harkness) to the challenge posed by brainstem surgery (Dr. Morota) and from spine and spinal cord tumor surgery (Dr. Hsu/Dr. Jallo, Dr. Drake) to the wide spectrum of pediatric neurosurgical procedures in the conus/ cauda region (Dr. Kothbauer/Dr. Deletis), a review on the state-of-the-art of intraoperative neurophysiology has been provided, with a focus on monitoring and mapping the motor system in children (Dr. Sala). The Letter to the Editor of Dr. Pang, appearing in this same issue of Child's Nervous System, comments Dr. Kothbauer's paper on neurophysiological monitoring for the lumbosacral nervous system. Dr. Pang has been using electrophysiology for many years to achieve total removal of complex spinal cord lipomas. His "wholehearted endorsement" of the neuromonitoring philosophy is one more evidence that, after all, intraoperative neurophysiology is here to stay.

\section{References}

1. Abramson J, Starfield B (2005) The effect of conflict of interest on biomedical research and clinical practice guidelines: can we trust the evidence in evidence-based medicine? J Am Board Fam Pract 18:414-418

2. Aghi M, Barker FG II (2006) Benign adult brain tumors: an evidence-based medicine review. In: Pollock BE (ed) Guiding neurosurgery by evidence. Karger, Basel, pp 80-96

3. Bagnall AM, Jones L, Duffy S, Riemsma RP (2008) Spinal fixation surgery for acute traumatic spinal cord injury. Cochrane Database Syst Rev, Issue 1. Art. No.: CD004725. doi:10.1002/14651858. CD004725.pub2

4. Bartholow R (1874) Experimental investigations into the functions of the human brain. Am J Med Sci 67:305-313

5. Boston JR, Deneault LG, Kronk L, Jannetta PJ (1985) Automated monitoring of brainstem auditory evoked potentials in the operating room. J Clin Monit 1:161-167

6. Daube J (1999) Intraoperative monitoring reduces complications and is therefore useful. Muscle and Nerve 22:1151-1153

7. Dinner DS, Luders H, Lesser RP, Morris HH, Barnett G, Klem G (1986) Intraoperative spinal somatosensory evoked potential monitoring. J Neurosurg 65:807-814

8. Fenn J, Raskino M (2008) Mastering the hype cycle. Harvard Business, Cambridge

9. Ginsburg HH, Shetter AG, Raudzens PA (1985) Postoperative paraplegia with preserved intraoperative somatosensory evoked potentials. J Neurosurg 63:296-300

10. Kombos T, Suess O, Brock M (2002) Cost analysis of intraoperative neurophysiological monitoring. Zentralbl Neurochir 63:141-145

11. Legatt AD, Emerson RG (2002) Motor evoked potential monitoring: it's about time. J Clin Neurophysiol 19:383-386

12. Lesser RP, Raudzens P, Lüders H, Nuwer MR, Goldie WD, Morris HH, Dinner DS, Klem G, Hahn JF, Shetter AG, Ginsburg HH, Gurd AR (1986) Postoperative neurological deficits may occur despite unchanged intraoperative somatosensory evoked potentials. Ann Neurol 19:22-25

13. Loewy EH (2007) Ethics and evidence-based medicine: is there a conflict? Med Gen Med 9:30

14. Malis L (1995) Intra-operative monitoring is not essential. Clin Neurosurg 42:203-213

15. Moeller A (1994) Intraoperative neurophysiological monitoring in neurosurgery: benefits, efficacy, and cost-effectiveness. Clin Neurosurg 42:171-179

16. Morledge DE, Stecker M (2006) The American Society of neurophysiological monitoring position statements project. J Clin Monit Comput 20:43-46

17. Nuwer MR (2002) Regulatory and medical-legal aspects of intraoperative monitoring. J Clin Neurophysiol 19:387-395 
18. Phillips LH II, Park TS (1990) Electrophysiological monitoring during lipomyelomeningocele resection. Muscle Nerve 13:127-132

19. Roos YB, Rinkel GJE, Vermeulen M, Algra A, van Gijn J (2003) Antifibrinolytic therapy for aneurysmal subarachnoid haemorrhage. Cochrane Database Syst Rev, Issue 2. Art. No.: CD001245. doi:10.1002/14651858.CD001245

20. Rosoff AJ (2001) Evidence-based medicine and the law: the courts confront clinical practice guidelines. J Health Polit Policy Law 26:327-368

21. Sahuquillo J, Arikan F (2006) Decompressive craniectomy for the treatment of refractory high intracranial pressure in traumatic brain injury. In: Cochrane Database Syst Rev, Issue 1. p Art. No.: CD003983. doi:003910.001002/14651858.CD14003983.pub 14651852
22. Sala F, Krzan MJ, Deletis V (2002) Intraoperative neurophysiological monitoring in pediatric neurosurgery: why, when, how? Childs Nerv Syst 18:264-287

23. Sekhar L (1994) Neurophysiological monitoring during cranial base surgery: is it necessary? Clin Neurosurg 42:180-202

24. Taniguchi M, Cedzich C, Schramm J (1993) Modification of cortical stimulation for motor evoked potentials under general anesthesia; technical description. Neurosurgery 32:219-226

25. Tator $\mathrm{CH}$ (2006) Review of treatment trials in human spinal cord injury: issues, difficulties, and recommendations. Neurosurgery 59:957-982, discussion 982-957

26. Whitfield PC, Kirkpatrick P (2001) Timing of surgery for aneurysmal subarachnoid haemorrhage. Cochrane Database Syst Rev, Issue 2. Art. No.: CD001697 